\title{
Simvastatin inhibits the adipogenesis of bone marrow-derived mesenchymal stem cells through the downregulation of chemerin/CMKLR1 signaling
}

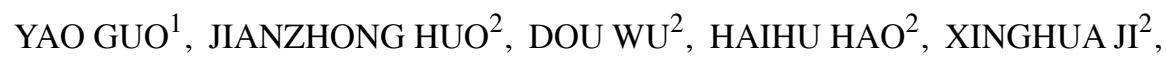 \\ ENZHE ZHAO ${ }^{1}$, BOYUAN NIE $^{3}$ and QIANG LIU ${ }^{2}$
}

\author{
${ }^{1}$ Shanxi Medical University, Taiyuan, Shanxi 030001; ${ }^{2}$ Department of Orthopaedics, Shanxi Bethune Hospital Affiliated \\ to Shanxi Medical University, Taiyuan, Shanxi 030032; ${ }^{3}$ Department of Orthopaedics, \\ Second Hospital of Shanxi Medical University, Taiyuan, Shanxi 030001, P.R. China
}

Received September 27, 2019; Accepted April 22, 2020

DOI: $10.3892 / \mathrm{ijmm} .2020 .4606$

\begin{abstract}
Simvastatin is effective in the treatment of osteoporosis, partly through the inhibition of the adipogenesis of bone-marrow derived mesenchymal stem cells (BMSCs). The present study focused on the mechanisms responsible for the inhibitory effects of simvastatin on adipogenesis and examined the effects of simvastatin on the expression of peroxisome proliferator-activated receptor $\gamma$ (PPAR $\gamma$ ), chemerin, chemokine-like receptor 1 (CMKLR1), G protein-coupled receptor 1 (GPR1) and the adipocyte marker gene, adiponectin. BMSCs were isolated from 4-week-old female Sprague-Dawley (SD) rats, and adipogenesis was measured by the absorbance values at $490 \mathrm{~nm}$ of Oil Red $\mathrm{O}$ dye. The expression of each gene was evaluated by western blot analysis or reverse transcription-quantitative PCR (RT-qPCR). The expression of chemerin increased during adipogenesis, while CMKLR1 exhibited a trend towards a decreased expression. On days 7 and 14 , the simvastatin-treated cells exhibited a downregulated expression of chemerin, whereas the upregulated expression of its receptor, CMKLR1 was observed. The results also revealed that CMKLR1 is required for adipogenesis
\end{abstract}

Correspondence to: Dr Qiang Liu, Department of Orthopaedics, Shanxi Bethune Hospital Affiliated to Shanxi Medical University, 99 Longcheng Street, Taiyuan, Shanxi 030032, P.R. China E-mail: sxdyy001@163.com

Abbreviations: BMSCs, bone marrow-derived mesenchymal stem cells; HSCs, hematopoietic stem cells; PPAR $\gamma$, peroxisome proliferator-activated receptor $\gamma$; CMKLR1, chemokine-like receptor 1; GPR1, G protein-coupled receptor 1; HMG-CoA reductase, 3-hydroxy-3-methyl-glutaryl-coenzyme A reductase; FPP, farnesyl pyrophosphate; GGPP, geranylgeranyl diphosphate; GGOH, geranylgeraniol

Key words: adipogenesis, bone marrow-derived mesenchymal stem cell, chemerin, CMKLR1, PPAR $\gamma$, simvastatin, osteoporosis, rosiglitazone and the simvastatin-mediated inhibitory effect on adipogenesis. Simvastatin regulated adipogenesis by negatively modulating chemerin-CMKLR1 signaling. Importantly, simvastatin stimulation inhibited the upregulation of PPAR $\gamma$ and PPAR $\gamma$-mediated chemerin expression to prevent adipogenesis. Treatment with the PPAR $\gamma$ agonist, rosiglitazone, partially reversed the negative regulatory effects of simvastatin. On the whole, the findings of the present study demonstrate that simvastatin inhibits the adipogenesis of BMSCs through the downregulation of PPAR $\gamma$ and subsequently prevents the PPAR $\gamma$-mediated induction of chemerin/CMKLR1 signaling.

\section{Introduction}

Osteoporosis is a systemic disease associated with decreased bone mass, leading to reduced bone quality and an increased risk of fracture (1) due to the functional imbalance of osteoclasts and osteoblasts (2). Osteoclasts and osteoblasts are derived from hematopoietic stem cells (HSCs) and bone marrow-derived mesenchymal stem cells (BMSCs) (3). In the bone marrow of patients with osteoporosis, the adipogenic differentiation of BMSCs prevails over osteogenic differentiation, leading to lipid accumulation $(4,5)$. An excessive amount of adipocytes in bone marrow may inhibit bone formation and stimulate bone resorption (6). Adipocytes may produce several adipokines such as leptin, adiponectin and chemerin (7) that alter bone matrix composition (8). Hence, inhibiting the adipogenesis of BMSCs may have the potential to increase osteogenesis.

Statins are normally used to lower cholesterol in patients with hyperlipidemia and cardiovascular diseases (9); however, they have also exhibited efficacy in the treatment of bone loss-related diseases (10-12). The liposoluble simvastatin exhibits anabolic effects on bones by stimulating osteogenesis, inhibiting osteoblasts apoptosis (13), and by suppressing the differentiation and activities of osteoclasts (13). Statins also increase the expression of the bone morphogenic protein-2, enhancing bone formation (14). An animal study using ovariectomized rats suggested that the use of low-dose statin increased bone resorption, while high-dose statin increased 
bone formation (15). Simvastatin has also been shown to negatively regulate the adipogenic differentiation of BMSCs and preadipocytes (16) through the downregulation of the peroxisome proliferator-activated receptor $\gamma(\operatorname{PPAR} \gamma)(16,17)$.

PPAR $\gamma$ activation favors BMSC differentiation into adipocytes, and the PPAR $\gamma$ blockade promotes osteogenesis (18). The overexpression of PPAR $\gamma$ and lipid accumulation have been found in the bone marrow from osteoporosis models (19). During adipocyte differentiation, PPAR $\gamma$ directly stimulates the expression and secretion of chemerin $(20,21)$. Chemerin binds to at least 3 receptors: Chemokine-like receptor 1 (CMKLR1), G protein-coupled receptor 1 (GPR1) and chemokine (C-C motif) receptor-like 2 (CCRL2) (22-24). CCRL2 is an atypical receptor and was not investigated in the present study. CMKLR1 and GPR1 are similar in sequence and structure; thus, GPR1 may partially mediate the function of chemerin (22-24). An increased level of chemerin or the deficiency of CMKLR1 leads to bone loss in mice $(25,26)$. Chemerin/CMKLR1 signaling is essential for adipogenesis $(20,27-29)$. On the other hand, the role of GPR1 in adipogenesis upon simvastatin treatment has been less extensively studied, despite a link between GPR1 and bone metabolism (30).

Nevertheless, the mechansims through which simvastatin inhibit adipogenesis remain unclear. In the present study, it was demonstrated that chemerin/CMKLR1 signaling drives the adipogenesis of BMSCs. Simvastatin downregulated the expression of chemerin, thereby inhibiting chemerin/CMKLR1 signaling and adipogenesis. It was also founde that PPAR $\gamma$ induced the expression of chemerin to activate downstream chemerin/CMKLR1 signaling. Simvastatin thus exerts its inhibitory effects by inhibiting PPAR $\gamma$ expression.

\section{Materials and methods}

Isolation, culture and identification of BMSCs. All animal experiments were performed in compliance with the research guidelines and were approved by the Ethics Committee of the Shanxi Medical University (no. 2017005). The rats were provided with free access to food and water. They were housed in a facility at $25^{\circ} \mathrm{C}, 75 \%$ humidity, with a $12-12 \mathrm{~h}$ light/dark cycle. BMSCs were isolated from one 4-week-old specific pathogen-free (SPF) female Sprague-Dawley (SD) rat (Vital River Laboratory), as previously described (30). The primary cells were cultured in minimum essential medium, $\alpha$-modification ( $\alpha$-MEM) containing $10 \%$ fetal bovine serum (FBS) and $1 \%$ penicillin-streptomycin solution in $75-\mathrm{cm}^{2}$ flasks. An atmosphere of $5 \% \mathrm{CO}_{2}$ and $37^{\circ} \mathrm{C}$ was maintained. The medium was changed every $72 \mathrm{~h}$ until $80 \%$ confluency was achieved. The BMSCs were trypsinized by treatment with $0.05 \%$ trypsin- $0.02 \%$ EDTA solution and passaged at a split ratio of 1:3. Once the same level of confluence was obtained, the cells at passage 3 were trypsinized and seeded at a density of $1.0 \times 10^{4}$ cells $/ \mathrm{cm}^{2}$ in $6-\mathrm{cm}$ dishes or 6 -well plates pre-coated with $0.1 \%$ gelatin solution.

According to the International Society for Cellular Therapy position statement (31), the identification of the cells was performed by examining the morphological features and flow cytometry, as previously described (30). The differentiation capacity of the BMSCs was also detected using SD rat bone mesenchymal stem cell osteogenic differentiation medium kit (RASMX-90021) and adipogenic differentiation medium kit (RASMX-90031), according to the manufacturer's instructions [Cyagen Biosciences (Guangzhou) Co., Ltd.].

Flow cytometry. Flow cytometry was performed according to a previously published method (30). BMSCs at passage 3 were trypsinized according to the method described above, and were then centrifuged at $179 \mathrm{x} \mathrm{g}$ for $4 \mathrm{~min}$ at room temperature. The cell concentration was then adjusted to $1.0 \times 10^{7}$ cells $/ \mathrm{ml}$. Subsequently, the cells were incubated with antibodies (BD Biosciences) against CD44 (FITC mouse anti-rat CD44, cat. no. 550974), CD45 (PE-Cy ${ }^{\mathrm{TM}} 5$ mouse anti-rat CD45, cat. no. 559135), CD90 (APC mouse anti-rat CD90, cat. no. 561409) and CD11b (PE mouse anti-rat CD11b, cat. no. 562105) at a concentration of $10 \mu \mathrm{l}$ of a $1 / 50$ dilution for $10^{6}$ cells in $100 \mu 1$. Following incubation for $30 \mathrm{~min}$ at $4^{\circ} \mathrm{C}$ protected from light, the samples were washed twice with wash buffer and resuspended in flow cytometry buffer. Fluorescence was measured using a FACSCalibur (BD Biosciences).

Proliferation analysis of BMSCs. The proliferation assay was performed using Cell Counting kit-8 (CCK-8) according to the manufacturer's instructions (AR1160, Wuhan Boster Biological Technology, Ltd.). Briefly, BMSCs were seeded in 96-well plates (1,000 cells/well) and cultured in the maintenance medium, which was changed every 3 days, with simvastatin (HY-17502, MedChemExpress) at various concentrations $\left(10^{-5} \mathrm{M}, 10^{-6} \mathrm{M}, 10^{-7} \mathrm{M}\right.$ and $\left.10^{-8} \mathrm{M}\right)$. PBS was added to the control group. The CCK-8 solution ( $10 \mu \mathrm{l} /$ well) was added at $1 \mathrm{~h}$ before absorbance values at $450 \mathrm{~nm}$ were read using a SPECTRA max PLUS microplate reader (Molecular Devices, LLC) to draw the growth curves.

Adipocyte differentiation. The conditioned media included basal media A and B. Basal medium A contained $\alpha$-MEM, $1 \%$ penicillin-streptomycin solution (Wuhan Boster Biological Technology, Ltd.), 10\% FBS (ScienCell Research Laboratories, Inc.), $10 \mu \mathrm{M}$ insulin, $200 \mu \mathrm{M}$ indomethacin, $500 \mu \mathrm{M}$ 3-isobutyl-1-methylxanthine and $1 \mu \mathrm{M}$ dexamethasone (Sigma-Aldrich; Merck KGaA). Basal medium B contained $\alpha$-MEM, FBS, penicillin-streptomycin solution and insulin at the same concentrations as in basal medium $\mathrm{A}$. The adipogenic induction of BMSCs followed a protocol for 3 cycles involving incubation in basal medium A for 3 days and then in basal medium B for 1 day. Subsequently, the cells were incubated in basal medium B until day 14 of differentiation. Moreover, to examine the role of PPAR $\gamma, 0.5 \mu \mathrm{M}$ rosiglitazone (HY-17386, MedChemExpress) was used as an agonist; thus, it was not included in the adipogenic induction medium.

Simvastatin was diluted beforehand with PBS and was added to the medium at a final concentration of $10^{-7} \mathrm{M}$ throughout the differentiation process. The conditioning medium of the control group contained the same amount of PBS. Oil Red O staining was performed at 14 days after induction to verify the effects of different intervention schemes of simvastatin (simvastatin incubation for 14 days; for the first 3 days; after 3 days) on adipogenesis. The adipogenic differentiated cells were washed twice with PBS, and then fixed with $2 \mathrm{ml}$ of $4 \%$ neutral formaldehyde (Boster Biological Technology) 
for $30 \mathrm{~min}$. The cells were washed again, and subsequently stained with filtered Oil Red O solution (60\% Oil Red O dye and $40 \%$ water) (Cyagen Biosciences, Inc.) at room temperature for $30 \mathrm{~min}$. The Oil Red $\mathrm{O}$ dye was further extracted from the adipogenic cells with $1 \mathrm{ml}$ isopropanol before absorbance values at $490 \mathrm{~nm}$ were read.

Adenovirus generation and transduction. CCX832, the only small-molecule antagonist for CMKLR1, is not commercially available (24). Therefore, the present study sought to knock down CMKLR1 (NM_008153) expression by RNA interference (RNAi). Virus packaging requires two types of plasmids. One is the packaging plasmid or auxiliary plasmid, which is used to express the capsid elements of the virus and to form the virus itself. The other is the shuttle vector, which is used to carry the shRNA expression system and is wrapped in the virus shell to form functional viral particles. An adenovirus backbone vector containing the target sequence 5'-GCAATG GCCTGGTGATTGTCA-3' was synthesized by GenePharma and co-transfected into 293A cells (Cell Bank of the Chinese Academy of Sciences) along with a shuttle vector, according to the manufacturer's instructions. The negative control carried a nonsense shRNA sequence (5'-GTT CTC CGA ACG TGT CAC GT-3'). This sequence does not target any gene in human, rat, or mouse. Adenovirus solution with a titer of $10^{8} \mathrm{TU} / \mathrm{ml}$ was prepared, and a multiplicity of infection of 100 was selected for subsequent BMSC transfection. The BMSCs were seeded onto 6-cm dishes (for western blot analysis) or 6-well plates [for reverse transcription-quantitative polymerase chain reaction (RT-qPCR)] $24 \mathrm{~h}$ prior to transfection. The adenovirus solution was then diluted using the transfection medium, which was $10 \%$ FBS and $5 \mu \mathrm{g} / \mathrm{ml}$ polybrene (Sigma-Aldrich; Merck KGaA) containing $\alpha$-MEM, and added into the culture dishes or plates. The transfection medium was replaced with culture medium after $24 \mathrm{~h}$. After a further $24 \mathrm{~h}$, the cell culture medium was replaced with the induction medium for differentiation. The differentiated cells were transfected using the adenovirus at 8 days post-differentiation with the same protocol to maintain interference efficiency. Adenovirus transfection efficiency was evaluated using an inverted fluorescence microscope (LX70; Olympus Corporation) by quantifying GFP expression. The interference efficiency was verified by the analysis of the mRNA and protein expression levels of CMKLR1.

Western blot analysis. For western blot analysis, proteins from the 14-days cultured cells of different groups were extracted using cell lysis buffer containing $1 \%$ phenylmethanesulfonyl fluoride and were centrifuged at $12,000 \mathrm{x} \mathrm{g}$ for $5 \mathrm{~min}$ at $4^{\circ} \mathrm{C}$. A BCA Protein Concentration Assay kit (AR0146, Wuhan Boster Biological Technology, Ltd.) was used to determine the protein concentration, according to the manufacturer's instructions. 10\% SDS-PAGE gel was used to separate the proteins, $50 \mu \mathrm{g} /$ lane of which was transferred onto a polyvinylidene difluoride membrane. The membrane was then incubated with primary antibodies for adiponectin (ab22554, mouse monoclonal antibody, 1:1,000 dilution, Abcam), CMKLR1 (ab64881, rabbit polyclonal antibody, 1:500 dilution, Abcam), $\beta$-actin (A5441, mouse monoclonal antibody, 1:10,000 dilution, Sigma-Aldrich; Merck KGaA) or $\beta$-tubulin (AF1216, rabbit monoclonal antibody, 1:10,000 dilution, Beyotime Institute of Biotechnology) overnight at $4^{\circ} \mathrm{C}$. The membrane was incubated with the appropriate secondary antibodies (adiponectin, A0216, horseradish peroxidase-conjugated goat anti-mouse IgG, 1:1,000 dilution, Beyotime Institute of Biotechnology; $\beta$-tubulin, A0208, horseradish peroxidase-conjugated goat anti-Rabbit IgG, 1:1,000 dilution, Beyotime Institute of Biotechnology; CMKLR1, 33101ES60, horseradish peroxidase-conjugated goat anti-rabbit $\mathrm{IgG}, 1: 2,000$ dilution, Yeasen Biotechnology (Shanghai) Co., Ltd.; $\beta$-actin: 33201ES60, horseradish peroxidase-conjugated goat anti-mouse IgG, 1:2,000 dilution, Yeasen Biotechnology (Shanghai) Co., Ltd.) for $2 \mathrm{~h}$ at room temperature. The specific blotting bands were detected by electrogenerated chemiluminescence and analyzed using a Gel-Pro Analyzer (Media Cybernetics). Protein expression was normalized to that of $\beta$-tubulin (Fig. 1) or $\beta$-actin (Fig. S4). Densitometry was performed using Gelpro32 4.0 software (Media Cybernetics).

Gene expression analysis. Gene expression analysis was performed using RT-qPCR. According to the manufacturer's instructions, RNAiso Plus (9108, Takara Bio, Inc.) was used to extract total RNA from the cultured cells of the different groups. The absorbance values at $260 \mathrm{~nm}$ were analyzed using a biospectrometer (Eppendorf AG) to determine the concentrations of extracted RNA, $0.4 \mu \mathrm{g}$ of which were subsequently mixed with 5X PrimeScript RT Master Mix (RR036A, Takara Bio, Inc.) and RNase-free $\mathrm{dH}_{2} \mathrm{O}$. The mixed solution $(10 \mu \mathrm{l})$ was used in reverse transcription reactions in a Veriti 96-Well Fast Thermal Cycler (Applied Biosystems) to generate cDNA. The cDNA solution was then mixed with SYBR Premix Ex Taq II (2X) (RR820A, Takara Bio, Inc.), primers $(0.4 \mu \mathrm{M})$, ROX Reference Dye, and RNase Free $\mathrm{dH}_{2} \mathrm{O}$ prior to quantitative polymerase chain reaction. mRNA expression analysis was performed using the StepOnePlus Real-Time PCR System (Applied Biosystems) in a two-step protocol according to the following conditions: Initial denaturation for $30 \mathrm{sec}$ at $95^{\circ} \mathrm{C}$, followed by 40 cycles with denaturation for $5 \mathrm{sec}$ at $95^{\circ} \mathrm{C}$, and annealing and extension for $30 \mathrm{sec}$ at $60^{\circ} \mathrm{C}$. All primer sequences are provided in Table I. The expression levels of PPAR $\gamma$, chemerin, CMKLR1, GPR1 and adiponectin were calculated relative to those of GAPDH by the $2^{-\Delta \Delta \mathrm{Cq}}$ method (32) for the 3, 7 and 14-day-differentiated cell samples. The melting curves obtained were examined for the specificity of the products prior to analysis.

Statistical analysis. Statistical analyses were performed using SPSS 22.0 (IBM Corp.). The results are expressed as the means \pm SD. A two-tailed Student's t-test was used to compare the mean values between the 2 groups. One-way analysis of variance (ANOVA) followed by the Dunnett or LSD post hoc tests was performed in the case of multiple comparisons. $\mathrm{P}<0.05$ was considered to indicate a statistically significant difference.

\section{Results}

Identification of BMSCs. To identify the BMSCs of SD rats, flow cytometry was performed and the presence of the BMSC marker proteins, CD44 and CD90, and the absence of the 
Table I. Primer sequences used for RT-qPCR.

\begin{tabular}{|c|c|c|}
\hline Gene & Primer sequence $\left(5^{\prime} \rightarrow 3^{\prime}\right)$ & Product size \\
\hline \multirow[t]{2}{*}{ GAPDH } & Fw: GATGCTGGTGCTGAGTATGT & $104 \mathrm{bp}$ \\
\hline & Rv: GCGGAGATGATGACCCTTT & \\
\hline \multirow[t]{2}{*}{ PPARG } & Fw: TCCCGTTCACAAGAGCTGAC & $107 \mathrm{bp}$ \\
\hline & Rv: ATAATAAGGCGGGGACGCAG & \\
\hline \multirow[t]{2}{*}{ RARRES2 } & Fw: GGTGTGGACAGTGCTGATGA & $169 \mathrm{bp}$ \\
\hline & Rv: TGGGGTCCAGTTTGATGCAG & \\
\hline \multirow[t]{2}{*}{ CMKLRI } & Fw: AGTGACTGATCAGCCGAGGA & $141 \mathrm{bp}$ \\
\hline & Rv: GATGTAGTCCGAGCCGTCAG & \\
\hline \multirow[t]{2}{*}{ GPRl } & Fw: CCGGACCCTGAAGAACTCAC & $86 \mathrm{bp}$ \\
\hline & Rv: CCCGGAAGTACAGGGTAGGA & \\
\hline \multirow[t]{2}{*}{$A D I P O Q$} & Fw: CAGCATTCAGCGTAGGGC & $191 \mathrm{bp}$ \\
\hline & Rv: GAAGAGGCTCACTTTCACATCC & \\
\hline
\end{tabular}

$G A P D H$, glyceraldehyde 3-phosphate dehydrogenase; $P P A R G$, peroxisome proliferator-activated receptor $\gamma$ gene; RARRES2, chemerin gene; $C M K L R 1$, chemokine-like receptor 1; GPR1, G protein-coupled receptor 1; $A D I P O Q$, adiponectin gene; Fw, forward; Rv, reverse.

monocyte marker, CD11b, or hematopoietic cell marker, CD45, were observed on the cell surface (Fig. 1A). The morphological features and differentiation capacity of the cultured cells were also examined and it was found that cultured BMSCs from passage 3 maintained a fibroblast-like morphology (Fig. 1B). The cells were incubated in a specific induction medium to induce their differentiation into osteoblasts or adipocytes. At 28 days post-osteogenic differentiation, calcium deposition was observed (Fig. 1B). Oil Red O staining at 21 days post-adipogenic differentiation revealed lipid accumulation within the cells (Fig. 1B). Taken together, these lines of evidence suggest that these cells were BMSCs and could be used for further experiments.

Determination of the concentration and intervention scheme of simvastatin. To determine whether various concentrations of simvastatin exert differential effects on cell growth, cell growth was monitored following simvastatin stimulation for 5 days. The results revealed that simvastatin slightly enhanced the proliferation of BMSCs at a low concentration $\left(10^{-8} \mathrm{M}\right)$, but inhibited cell growth at higher concentrations $\left(10^{-5} \mathrm{M}\right.$ and $10^{-6} \mathrm{M}$ ) (Fig. 1C). Cells treated with $10^{-7} \mathrm{M}$ simvastatin exhibited a similar growth pattern compared to the control group (Fig. 1C).

To verify the intervention scheme of simvastatin on adipogenesis, Oil Red O staining of adipogenic BMSCs treated with simvastatin for 14 days (SIM group) was performed. In addition, BMSCs were examined during the first 3 days (group A, early stage of adipogenesis) and after 3 days (group B) of adipogenesis; BMSCs treated with PBS (CON group) were also examined. The protein expression of adiponectin in the simvastatin-treated group was markedly decreased compared to that of the control group, and the lipid accumulation within the cells exhibited a similar simvastatin-dependent lipid loss (Fig. 1D and E), indicating that simvastatin inhibited the adipogenic differentiation of BMSCs during the whole process of adipogenesis. Moreover, the simvastatin-treated group (SIM group) exhibited a lower absorbance at $490 \mathrm{~nm}$ compared with the control group or group A (Fig. 1F). The SIM group exhib- ited a comparable level of absorbance with group B (Fig. 1F). In addition, the fact that the inhibitory effect was more potent in group B and the SIM group than in group A (Fig. 1F) indicated that longer stimulation with simvastatin enhanced its inhibitory effects. The P-values obtained for the data in Fig. 1 are provided in detail in Tables SI and SII.

Effects of simvastatin on PPAR $\gamma$, chemerin/CMKLR1 signaling and adiponectin expression. In agreement with previous studies, the expression of chemerin increased after 3 , 7 and 14 days of adipogenesis, while one of the receptors for chemerin, CMKLR1, exhibited a trend towards a decreased expression (data not shown).

Simvastatin treatment did not affect the overall kinetics of the expression patterns and exerted no marked effects on the expression of chemerin and CMKLR, compared to the control group on day 3 (Fig. 2A and B). On days 7 and 14, the simvastatin-treated cells exhibited a downregulated expression of chemerin, whereas they exhibited an upregulated expression of its receptor, CMKLR1 (Fig. 2A and B). Notably, the expression of the adipocyte marker, adiponectin, was decreased compared with the control at day 14 (Fig. 2C), suggesting that simvastatin exerted an inhibitory effect on adipogenesis. PPAR $\gamma$ expression increased during adipogenesis while the expression of the other chemerin receptor, GPR1, exhibited a decreasing trend (data not shown). Simvastatin treatment decreased PPAR $\gamma$ expression on day 3 (Fig. S1). Simvastatin treatment did not affect the expression of GPR1 on days 3 and 7, but induced GPR1 expression on day 14 (Fig. S2A). The P-values for the data in Fig. 2 are provided in detail Tables SIII-SV.

CMKLR1 is required for adipogenesis and the simvastatin-mediated inhibitory effect on adipogenesis. Since simvastatin was found to differentially regulate the expression of chemerin and its receptor CMKLR1 in BMSCs, the present study directly examined the role of chemerin/CMKLR1 signaling during the simvastatin-mediated inhibition of adipogenesis by knocking down CMKLR1 in BMSCs using 

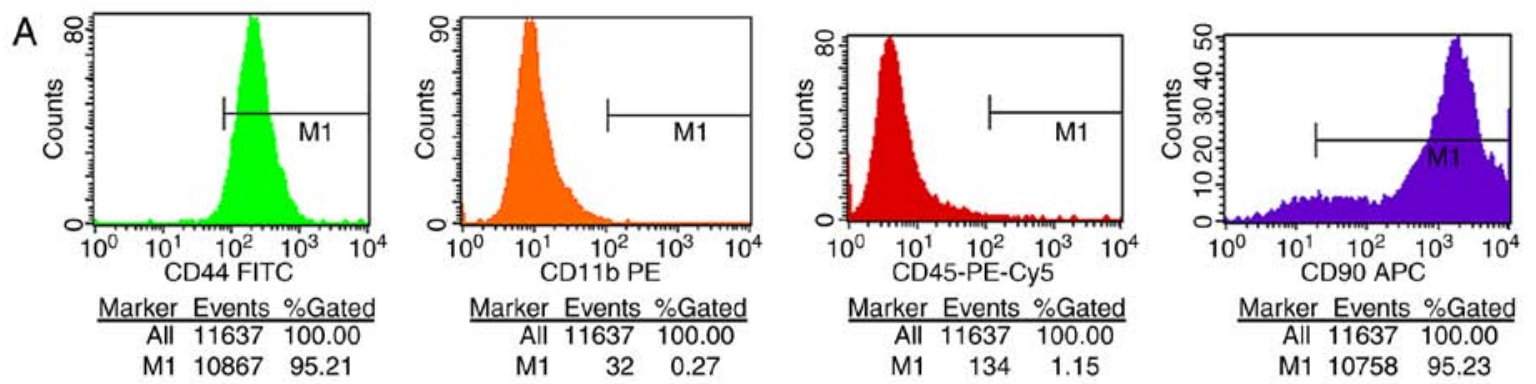

B
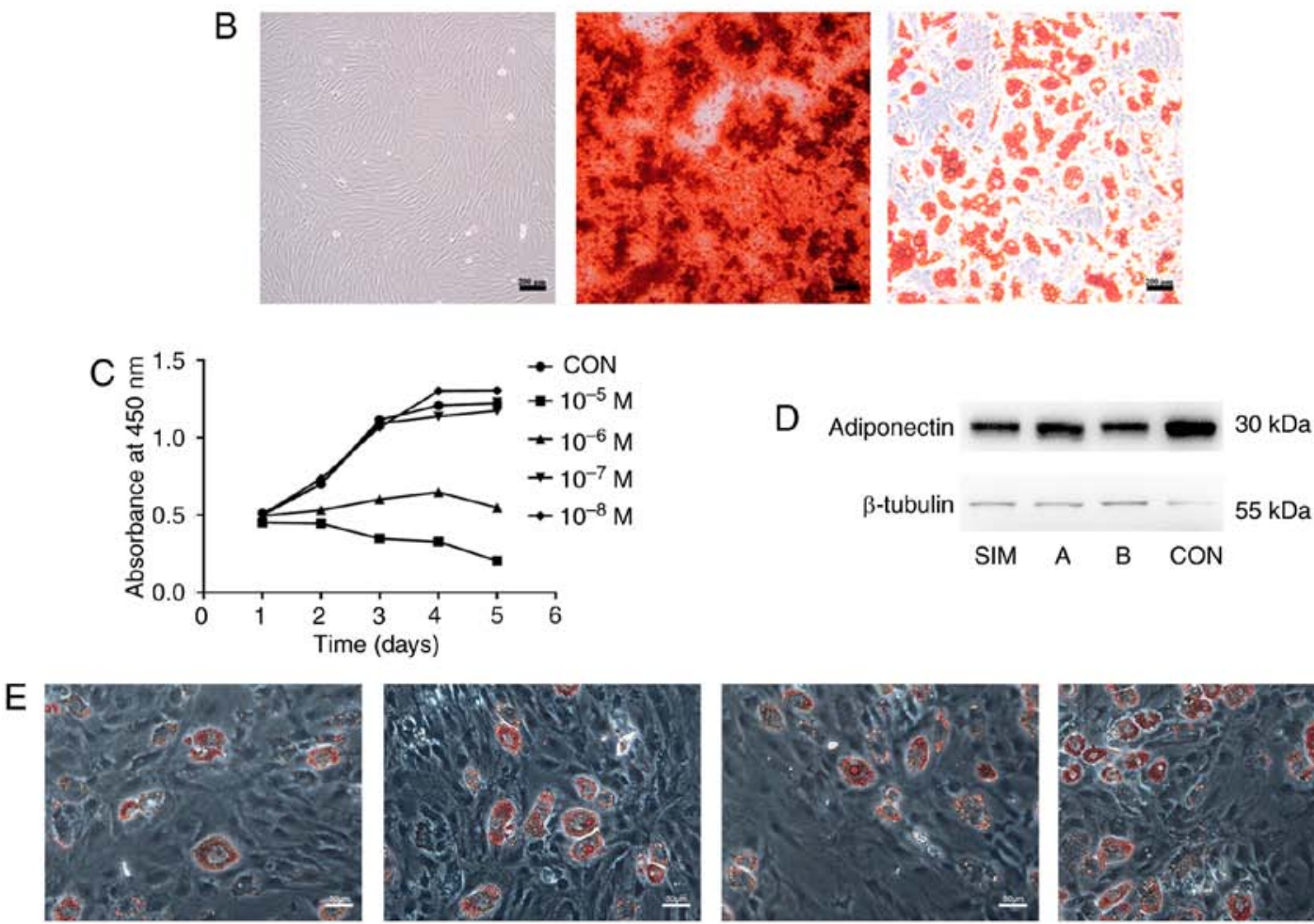

$\operatorname{SIM}$

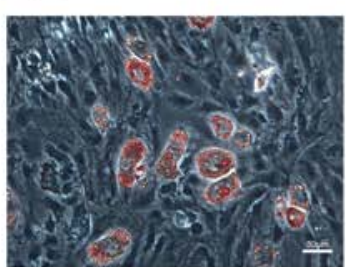

A

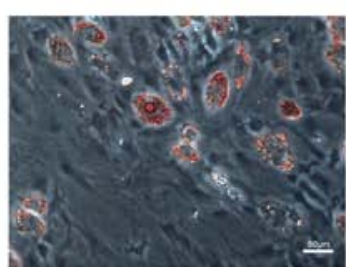

B

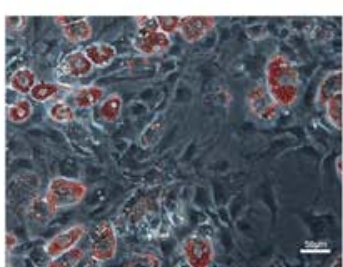

CON

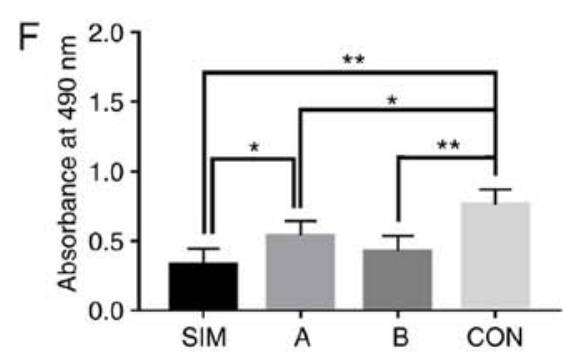

Figure 1. Determination of the concentration and scheme of simvastatin stimulation. (A) Prevalence and number of cells expressing the cell surface marker CD44, CD90, CD11b, or CD45 examined by flow cytometry. (B) Representative images of BMSCs from passage 3 under a light microscope (left panel), Alizarin Red staining after osteogenic induction (middle panel) and Oil Red O staining following adipogenic induction (right panel) (magnification, x100). (C) Growth curves of BMSCs treated with simvastatin at various concentrations or PBS ( $=5$ ). Data were examined by analysis of variance followed by Dunnett's post hoc test. (D) Western blot analysis of adiponectin levels. (E) Representative images of Oil Red O staining on differentiated BMSCs from different groups (magnification, x200). (F) Absorbance values (n=3) at $490 \mathrm{~nm}$ of cells as in (E). The data are expressed as the means \pm SD. Data were examined by analysis of variance followed by the LSD post hoc test. ${ }^{*} \mathrm{P}<0.05$ and ${ }^{* *} \mathrm{P}<0.01$. BMSCs, bone marrow-derived mesenchymal stem cells; SIM, BMSCs treated with simvastatin for 14 days; A, for the first 3 days; B, after 3 days; and CON, with PBS for 14 days.

RNAi. BMSCs were transfected with adenovirus at $48 \mathrm{~h}$ prior to adipogenic differentiation, and the immunofluorescence of each group was confirmed (Fig. S3). In comparison with the control group, a markedly lower expression of CMKLR1 at the protein (Fig. S4) and mRNA (Fig. 3A) level was detected on day 3 post-differentiation. The expression of GPR1, which shares sequence and structural similarities with CMKLR1, was also markedly decreased on days 3 and 14 post-differentiation (Fig. S2B), while a compensatory increase was not observed. Of note, the knockdown of CMKLR1 markedly inhibited adipogenesis, as evidenced by the decreased expression of adiponectin at 14 days post-differentiation (Fig. 3B). Consistently, decreased Oil Red O staining was observed in the cells in which CMKLR1 was knocked down (Fig. 3C), 

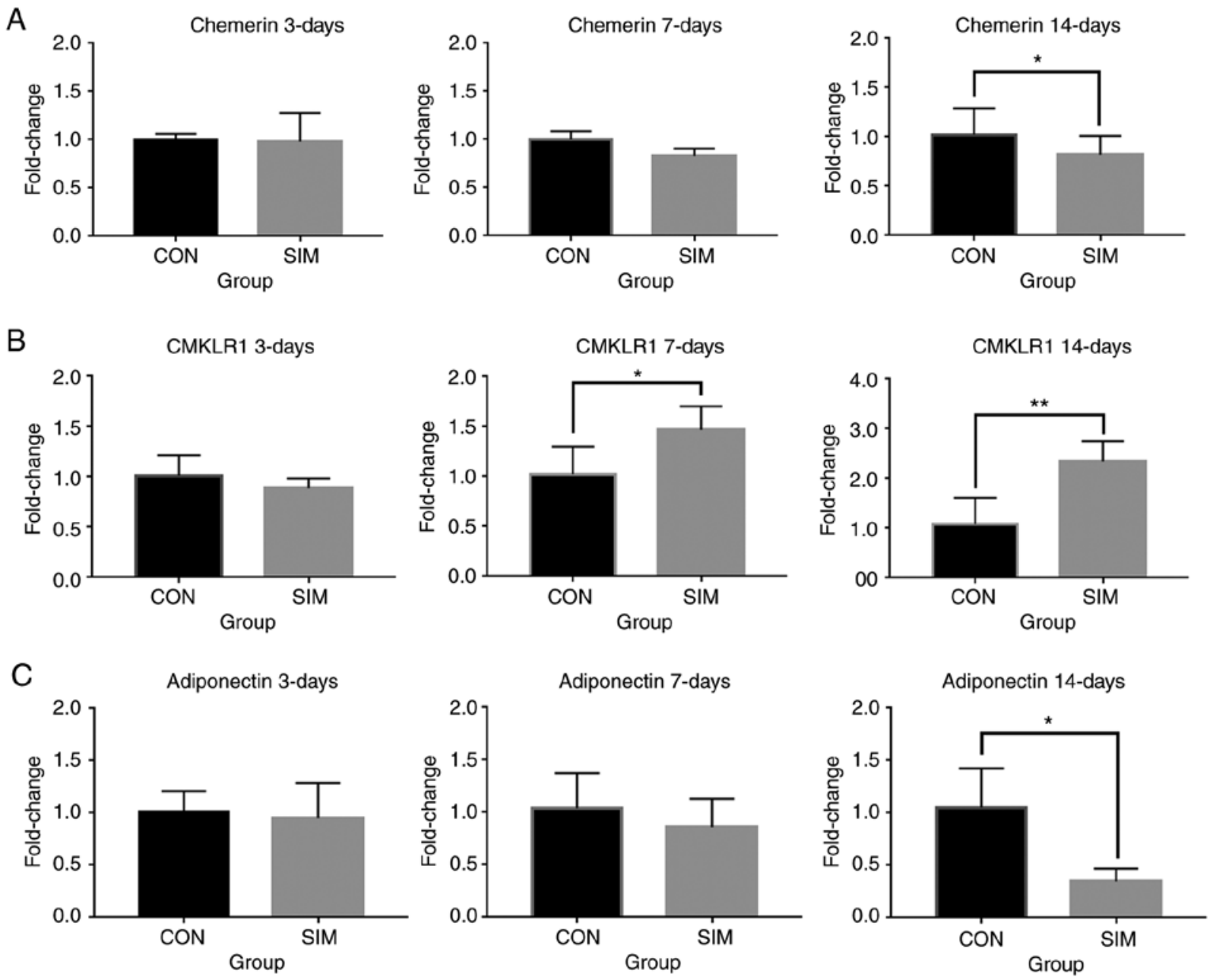

Figure 2. Effects of simvastatin on gene expression in BMSCs during adipogenic differentiation. Expression levels of (A) chemerin, (B) CMKLR1, and (C) adiponectin after simvastatin (SIM) or PBS (CON) treatment at days 3, 7 and 14 post differentiation analyzed by RT-qPCR (n=3). All RT-qPCR assays were performed 3 times and in triplicate. Fold change was expressed relative to the CON group, and the data are expressed as the means \pm SD. Data were analyzed by a Student's t-test. ${ }^{*} \mathrm{P}<0.05$ and ${ }^{* *} \mathrm{P}<0.01$. BMSCs, bone marrow-derived mesenchymal stem cells; CMKLR1, chemokine-like receptor 1 .

which was further confirmed by quantitative analysis (Fig. 3D), suggesting that the inhibition of chemerin/CMKLR1 signaling prevented the adipogenesis of BMSCs. Notably, simvastatin stimulation did not further decrease the expression of adiponectin, or Oil red $\mathrm{O}$ staining in the cells in which CMKLR1 was knocked down (Fig. 3C and D), suggesting that the inhibitory effects of simvastatin on adipogenesis of BMSCs are dependent on chemerin/CMKLR1 signaling. This is consistent with the negative regulation of chemerin expression by simvastatin stimulation observed above (Fig. 2), further supporting the notion that simvastatin negatively regulates adipogenesis by modulating chemerin/CMKLR1 signaling. The P-values for the data presented in Fig. 3 are provided in detail in Tables SVI-SVIII.

PPAR $\gamma$ agonist, rosiglitazone, partially reverses the effects of simvastatin on the expression of specific genes. To further determine whether PPAR $\gamma$ targets chemerin/CMKLR1 signaling during the simvastatin-mediated inhibition, the cells were treated with the PPAR $\gamma$ agonist, rosiglitazone. On days 3 and 14, rosiglitazone significantly increased the expression of PPAR $\gamma$ (Fig. 4A) and chemerin (Fig. 4B), suggesting that PPAR $\gamma$ indeed activates chemerin/CMKLR1 signaling downstream. Moreover, rosiglitazone also partially reversed the downregulation of PPAR $\gamma$ and chemerin induced by simvastatin on days 3 and 14 (Fig. 4A and B). The expression of CMKLR1 and the other receptor GPR1 were decreased by treatment with rosiglitazone (Figs. 4C and S2C). Consistent with an increase in the expression of chemerin, an increase in adipogenesis was observed in the rosiglitazone-treated BMSCs following simvastatin stimulation, as evidenced by the higher expression of adiponectin at 14 days compared to the control group (Fig. 4D). The P-values for the data presented in Fig. 4 are provided in detail in Tables SIX-SX.

Taken together, these data suggest that simvastatin inhibits PPAR $\gamma$-mediated chemerin signaling to prevent adipogenesis in BMSCs and that the activation of PPAR $\gamma$ signaling by rosiglitazone is able to reverse, at least partially, such an inhibitory effect.

\section{Discussion}

In addition to clinical applications for the treatment of hyperlipidemia and cardiovascular diseases, statins reduce the production of mevalproic acid and subsequent downstream products involved in various physiological and pathological 

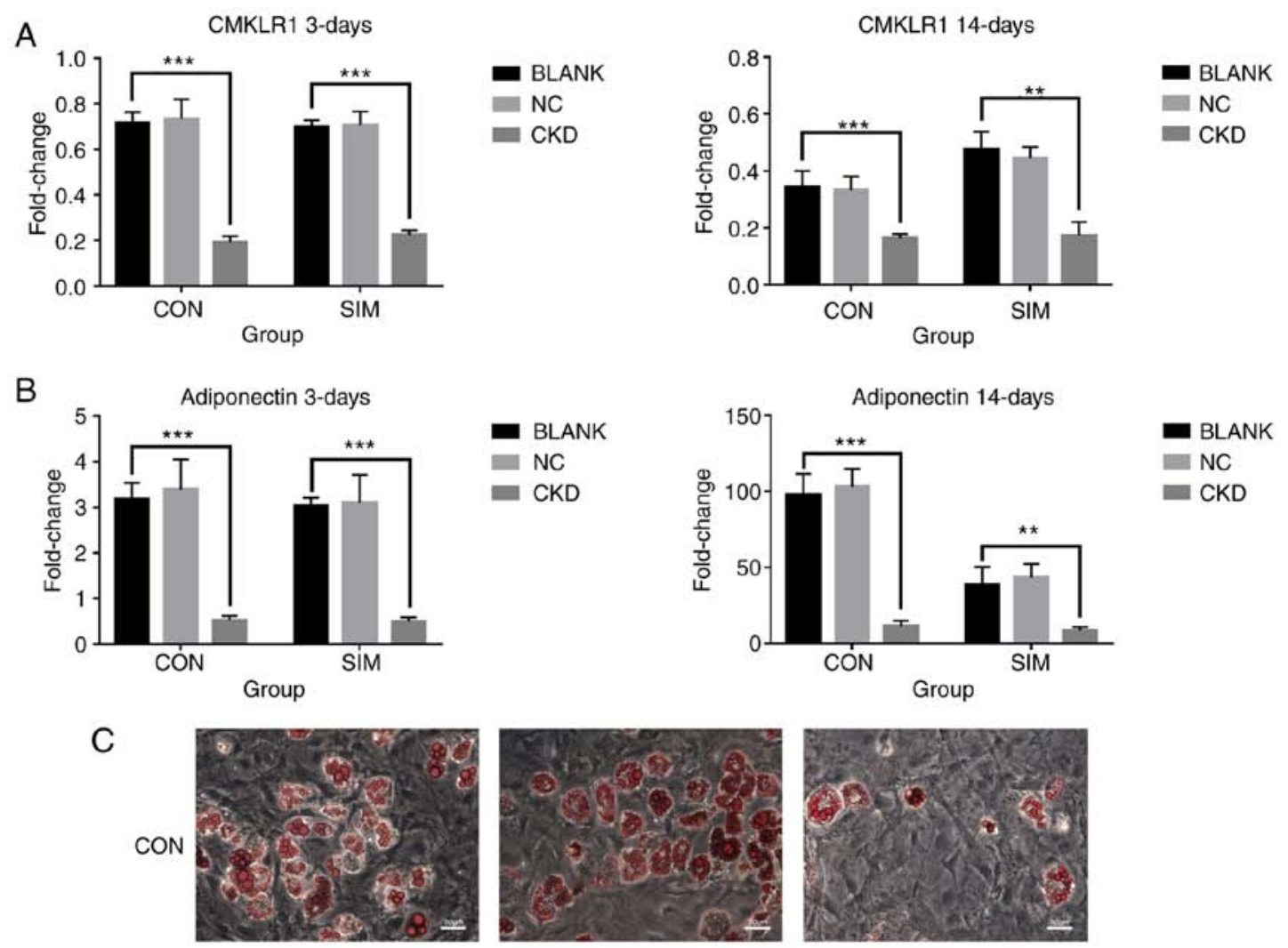

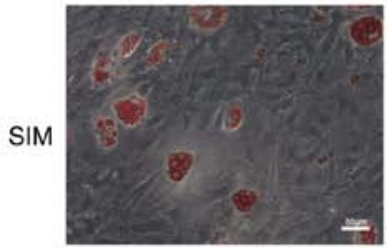

BLANK

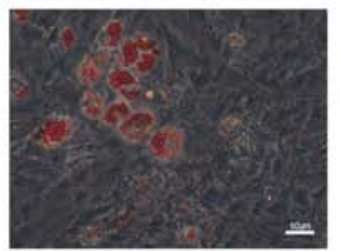

NC

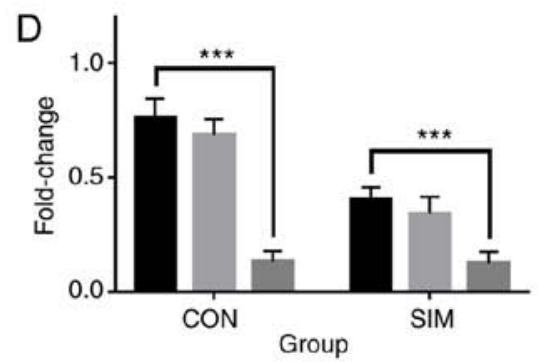

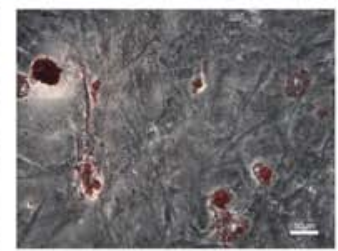

CKD

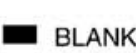

NC

- CKD

Figure 3. CMKLR1 is required for driving adipogenesis. BMSCs were transfected with or without adenovirus $48 \mathrm{~h}$ before differentiation. At days 3 and 14 post-differentiation, cells that exhibited adipogenic differentiation with PBS (CON) or simvastatin (SIM) treatment were analyzed by RT-qPCR for (A) CMKLR1, and (B) adiponectin expression ( $\mathrm{n}=3$ ). Fold change was expressed relative to undifferentiated BMSCs. (C) Representative images of Oil red $\mathrm{O}$ staining on BMSCs at days 14 post-differentiation from different groups (magnification, $x 200)$. (D) Absorbance values $(\mathrm{n}=3)$ at $490 \mathrm{~nm}$ of cells as in $(C)$. The data are expressed as the means $\pm \mathrm{SD}$. Data were examined by analysis of variance followed by the LSD post hoc test. ${ }^{* *} \mathrm{P}<0.01$ and ${ }^{* * *} \mathrm{P}<0.001$. BMSCs, bone marrow-derived mesenchymal stem cells; CMKLR1, chemokine-like receptor 1; BLANK, untransfected cells; NC, negative control; CKD, CMKLR1 knockdown.

processes (33). These are the so-called statin pleiotropic effects, one of which is on adipogenesis through the chemerin signaling $(29,34)$. Other examples are that statins may activate Wnt/ $\beta$-catenin signaling $(35,36)$ and inhibit Rho $(37,38)$ in different biological processes, and coincidentally, both CMKLR1 and GPR1 mediate chemerin signaling through the RhoA/ROCK pathway (39), and the former has been proven to be a Wnt target gene (27). Thus, the regulation of chemerin signaling via multiple mechanisms may, at least partly, account for the pleiotropic effects of statins. Nevertheless, although this coincidence indicates a possible link between chemerin signaling and statins, no direct evidence has been found to date. To the best of our knowledge, the present study is the first to bridge the inhibitory effect of simvastatin on adipogenesis of BMSCs and chemerin signaling through the regulation of PPAR $\gamma$ (Fig. 5).

Owing to evidence that the post-confluence clone expansion of BMSCs may influence adipogenesis (40), and 
A

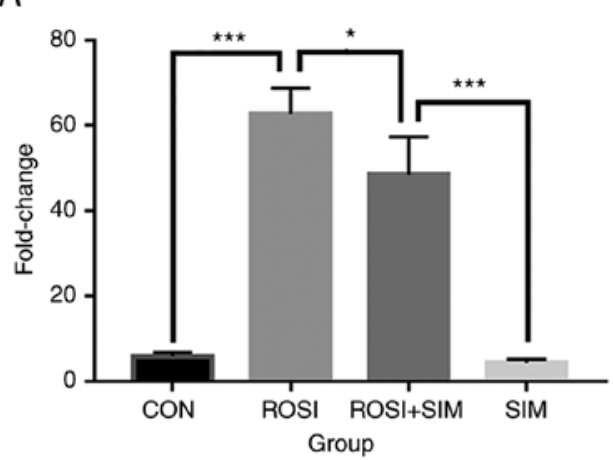

B

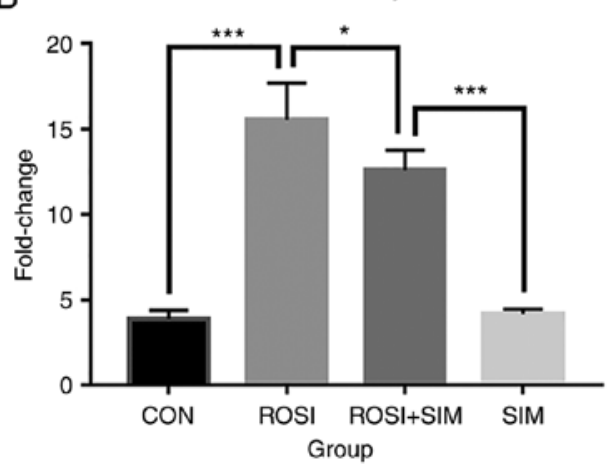

C

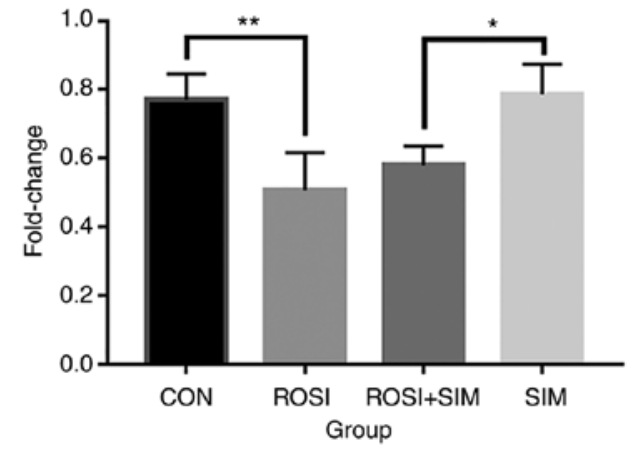

D

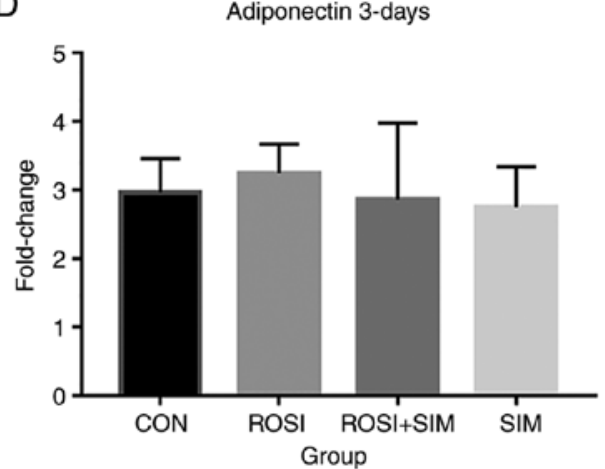

PPAR 14-days
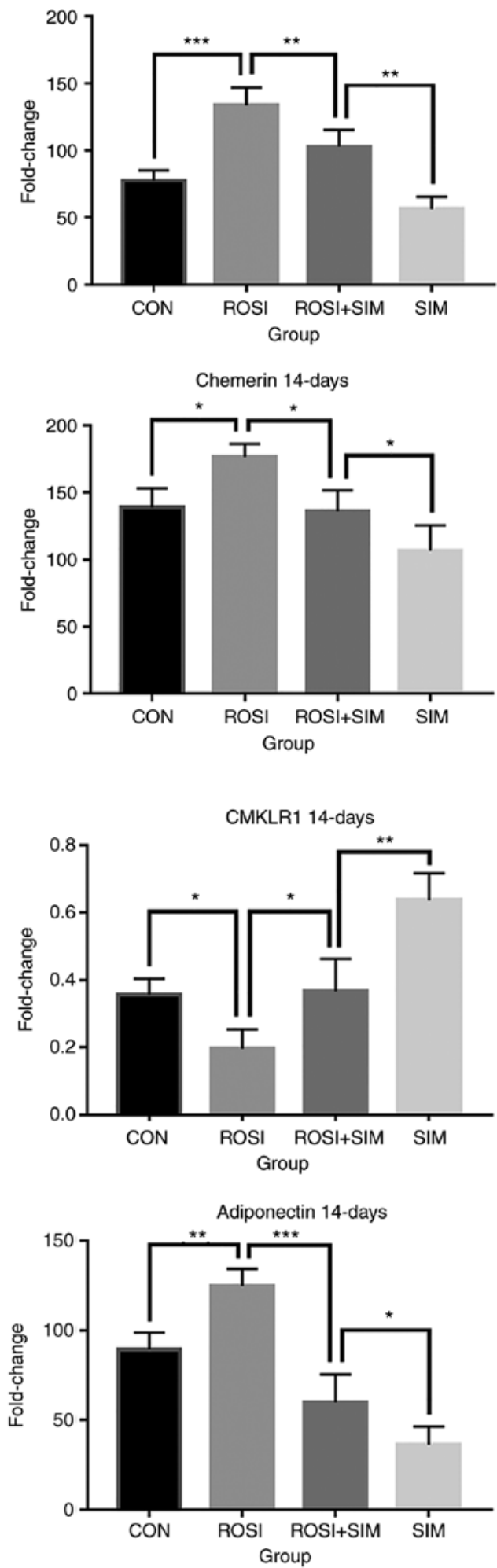

Figure 4. PPAR $\gamma$ agonist rosiglitazone partly reverses the effects of simvastatin on the expression of specific genes. At days 3 and 14 post-differentiation, cells that exhibited adipogenic differentiation with PBS (CON), rosiglitazone (ROSI), rosiglitazone and simvastatin (ROSI + SIM) and simvastatin (SIM) treatment were analyzed by RT-qPCR for expression of (A) PPAR $\gamma,(B)$ chemerin, (C) CMKLR1, and (D) adiponectin ( $\mathrm{n}=3$ ). Fold change was expressed relative to undifferentiated BMSCs, and the data are expressed as the means \pm SD. Data were examined by analysis of variance followed by the LSD post hoc test. ${ }^{*} \mathrm{P}<0.05,{ }^{* *} \mathrm{P}<0.01$ and ${ }^{* * * *} \mathrm{P}<0.001$. BMSCs, bone marrow-derived mesenchymal stem cells; CMKLR1, chemokine-like receptor 1 ; PPAR $\gamma$, peroxisome proliferator-activated receptor $\gamma$.

that simvastatin had effects, though with controversy, on BMSC proliferation $(41,42)$, the present study selected the concentration of simvastatin which minimally influenced proliferation to minimize its effect on proliferation-related adipogenesis. Furthermore, although the early stage is of vital importance for adipogenesis (28), simvastatin intervention was 


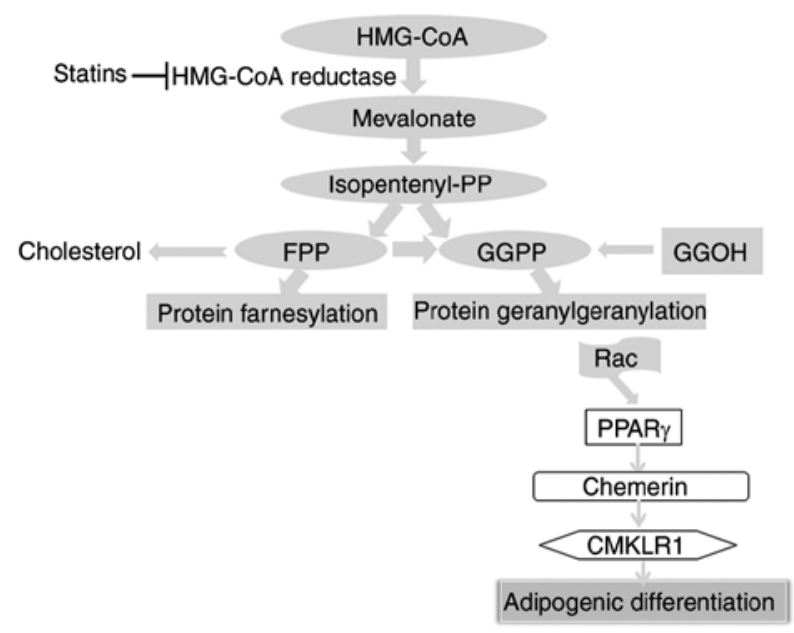

Figure 5. Summary of the effects of statins on adipogenic differentiation. The present study provides an explanation of the bridge between the inhibitory effect of simvastatin and adipogenesis in BMSCs and chemerin signaling through the regulation of PPAR $\gamma$. Simvastatin cannot affect adipogenesis without functional CMKLR1. Hence, CMKLR1 mediates the downregulated signals of PPAR $\gamma$ and chemerin in the process of simvastatin-mediated inhibition of adipogenesis. HMG-CoA, 3-hydroxy-3-methyl-glutaryl-coenzyme A reductase; FPP, farnesyl pyrophosphate; GGPP, geranylgeranyl diphosphate; GGOH, geranylgeraniol; PPAR $\gamma$, peroxisome proliferator-activated receptor $\gamma$; CMKLR1, chemerin chemokine-like receptor 1.

performed in the whole adipogenic differentiation process of BMSCs to maintain its continuous role in adipogenesis and its possible role on the expression of PPAR $\gamma$ and chemerin signaling. It has been reported that the decreased expression of PPAR $\gamma$ leads to the simvastatin-mediated inhibition of adipogenesis $(43,44)$, and chemerin, which plays vital roles in adipogenesis, was a target gene of PPAR $\gamma$ in adipogenesis of BMSCs (20). The results of the present study demonstrated that the expression of both genes increased significantly during the adipogenic differentiation process, and simvastatin intervention inhibited such increase, demonstrating that the inhibitory effect of simvastatin may be due to the downregulation of PPAR $\gamma$ and chemerin signals.

It is noteworthy that while the chemerin receptor CMKLR1 expression was upregulated by simvastatin, chemerin-mediated adipogenesis was still inhibited by simvastatin. This may be due to that the decreased expression of chemerin by simvastatin stimulation overrides the elevated expression of CMKLR1, thereby leading to the inhibition of chemerin-CMKLR1 signaling and reduced adipogenesis. Similarly, the PPAR $\gamma$ agonist rosiglitazone partially rescued the inhibitory effect of simvastatin but reduced the levels of CMKLR1. In this case, the upregulated expression of chemerin by PPAR $\gamma$ activation may override CMKLR1 downregulation leading to increased chemerin-CMKLR1 signaling and adipogenesis. Indeed, PPAR $\gamma$ has been shown to decrease $\beta$-catenin-dependent CMKLR1 expression by promoting the degradation of $\beta$-catenin (29). Moreover, consistent with the findings of the present study, the downregulation of CMKLR1 is associated with an enhancement of chemerin/CMKLR1 signaling leading to bone resorption activity in mature osteoclasts (28).

Apart from the expression of PPAR $\gamma$, an increase in chemerin expression was also detected by rosiglitazone inter- vention in the present study, and a possible role of PPAR $\gamma$ in the simvastatin-mediated inhibition of adipogenesis was demonstrated. Moreover, the decreased expression of both chemerin receptors, CMKLR1 and GPR1, along with adipogenesis, was further reduced in the context of rosiglitazone, and such a decrease was partially reversed by simvastatin intervention. To further validate this hypothesis, RNAi was used to knock down the expression of CMKLR1. Simvastatin treatment did not exert a further inhibition of adipogenesis with CMKLR1 knockdown; in other words, simvastatin intervention did not affect adipogenesis without functional CMKLR1. Hence, CMKLR1 mediates the downregulated signals of PPAR $\gamma$ and chemerin in the process of simvastatin-mediated inhibition of adipogenesis (Fig. 5).

Considering the controversies in functional studies of CMKLR1, researchers have proposed that the functional differences of CMKLR1 may partlly be due to the activation of GPR1 by chemerin (28). The results of the present study demonstrated a similar decrease in the gene expression levels of CMKLR1 and GPR1 during adipogenesis, and the decreased expression of both receptors was inhibited by simvastatin, thus suggesting that GPR1 may function similarly as CMKLR1 during this process. Nevertheless, although both receptors can modulate the osteogenic differentiation of BMSCs, only CMKLR1 was proven to be effective in adipogenesis. Surprisingly, it was found that GPR1 expression markedly decreased after CMKLR1 knockdown, and this decrease was not promoted by simvastatin. These results indicate that in the absence of CMKLR1, GPR1 may not significantly affect the inhibitory effect of simvastatin, and the increase in the expression of GPR1 with simvastatin may simply be due to the inhibitory effect of simvastatin on adipogenesis. Moreover, GPR1 showed lower expression than CMKLR1, demonstrating that the function of GPR1 may not be as important as that of CMKLR1 during adipogenesis. This result was in accordance with the findings of the study by Rourke et al, that GPR1 did not contribute to adipogenesis (45). Despite the expression data on GPR1 from the present study, the role of this receptor in the adipogenesis of BMSCs remains to be further revealed.

In the present study, the effects of simvastatin on chemerin and CMKLR1 expression were not verified at the protein level, and translation, post-translation processing and modification, and different detection times may lead to inconsistent mRNA and protein levels. It is not known whether the lipid-inhibiting effect of simvastatin is directly achieved through the chemerin and CMKLR1 proteins, or whether it affects proteins downstream of the chemerin signaling pathway. This is a limitation of the present study. Therefore, the expression of chemerin and CMKLR1 at the protein level may or may not be consistent with the changes at the RNA level. To solve the problem of gene expression differences at different levels, well-designed experiments are required to eliminate the interference of various factors in gene translation.

In conclusion, for the first time, to the best of our knowledge, the present study demonstrates that PPAR $\gamma$ bridges the inhibitory effect of statins on adipogenesis and chemerin signaling. Simvastatin inhibits the adipogenesis of BMSCs through the downregulation of PPAR $\gamma$ and subsequently prevents PPAR $\gamma$-mediated induction of chemerin/CMKLR1 signaling. This partially explains how simvastatin inhibits the 
adipogenesis of BMSCs and provides new ideas for the clinical application of statins on osteoporosis treatment. Considering that there may be more possible connections between chemerin signaling and the pleiotropic effects of statins, more information about the relevant downstream events must be obtained to have a better understanding of their interplay.

\section{Acknowledgements}

Not applicable.

\section{Funding}

The present study was supported by the Shanxi Province Science and Technology Research Project (grant no. 20150313012-1); and Key Research and Development (R\&D) Projects of Shanxi Province (grant no. 201803D31134).

\section{Availability of data and materials}

The datasets used and/or analyzed during the current study are available from the corresponding author on reasonable request.

\section{Authors' contributions}

YG was the main contributor to the conception and design of the present study and in the drafting of the manuscript. JH and DW played an important role in data analysis and contributed to the writing of the manuscript. $\mathrm{HH}$ and $\mathrm{XJ}$ performed the experiments of cell culture, cell identification and RNAi. EZ and BN performed RT-qPCR and western blot analysis, and analyzed the collected data. QL played an important role in the conception and design of the study, and revised the manuscript. All authors read and approved the final manuscript.

\section{Ethics approval and consent to participate}

All animal experiments were performed in compliance with the research guidelines and approved by the Ethics Committee of the Shanxi Medical University (no. 2017005).

\section{Patient consent for publication}

Not applicable.

\section{Competing interests}

The authors declare that they have no competing interests.

\section{References}

1. Kanis JA, McCloskey EV, Johansson H, Oden A, Melton LJ III and Khaltaev N: A reference standard for the description of osteoporosis. Bone 42: 467-475, 2008.

2. Teitelbaum SL: Bone resorption by osteoclasts. Science 289 : 1504-1508, 2000.

3. Marks SC Jr: The origin of osteoclasts: Evidence, clinical implications and investigative challenges of an extra-skeletal source. J Oral Pathol 12: 226-256, 1983

4. Chamberlain G, Fox J, Ashton B and Middleton J: Concise review: Mesenchymal stem cells: Their phenotype, differentiation capacity, immunological features, and potential for homing. Stem Cells 25: 2739-2749, 2007.
5. Bianco P, Robey PG, Saggio I and Riminucci M: 'Mesenchymal' stem cells in human bone marrow (skeletal stem cells): A critical discussion of their nature, identity, and significance in incurable skeletal disease. Hum Gene Ther 21: 1057-1066, 2010.

6. Muruganandan S and Sinal CJ: The impact of bone marrow adipocytes on osteoblast and osteoclast differentiation. IUBMB Life 66: 147-155, 2014.

7. de Paula FJA and Rosen CJ: Structure and function of bone marrow adipocytes. Compr Physiol 8: 315-349, 2017.

8. Mauney $\mathbf{J}$ and Volloch V: Progression of human bone marrow stromal cells into both osteogenic and adipogenic lineages is differentially regulated by structural conformation of collagen I matrix via distinct signaling pathways. Matrix Biol 28: 239-250, 2009.

9. Anderson TJ, Grégoire J, Pearson GJ, Barry AR, Couture P, Dawes M, Francis GA, Genest J Jr, Grover S, Gupta M, et al: 2016 Canadian cardiovascular society guidelines for the management of dyslipidemia for the prevention of cardiovascular disease in the adult. Can J Cardiol 32: 1263-1282, 2016.

10. Shah SR, Werlang CA, Kasper FK and Mikos AG: Novel applications of statins for bone regeneration. Natl Sci Rev 2: 85-99, 2015.

11. Lin TK, Chou P, Lin CH, Hung YJ and Jong GP: Long-term effect of statins on the risk of new-onset osteoporosis: A nationwide population-based cohort study. PLoS One 13: e0196713, 2018.

12. Lin TK, Liou YS, Lin CH, Chou P and Jong GP: High-potency statins but not all statins decrease the risk of new-onset osteoporotic fractures: A nationwide population-based longitudinal cohort study. Clin Epidemiol 10: 159-165, 2018.

13. Moshiri A, Sharifi AM and Oryan A: Role of Simvastatin on fracture healing and osteoporosis: A systematic review on in vivo investigations. Clin Exp Pharmacol Physiol 43: 659-684, 2016.

14. Jadhav SB and Jain GK: Statins and osteoporosis: New role for old drugs. J Pharm Pharmacol 58: 3-18, 2006.

15. Maritz FJ, Conradie MM, Hulley PA, Gopal R and Hough S: Effect of statins on bone mineral density and bone histomorphometry in rodents. Arterioscler Thromb Vasc Biol 21: 1636-1641, 2001.

16. Bajaj MS, Kulkarni RS, Ghode SS, Limaye LS and Kale VP: Irradiation-induced secretion of BMP4 by marrow cells causes marrow adipogenesis post-myelosuppression. Stem Cell Res 17: 646-653, 2016.

17. Liu M, Wang K, Tang T, Dai K and Zhu Z: The effect of simvastatin on the differentiation of marrow stromal cells from aging rats. Pharmazie 64: 43-48, 2009.

18. Yuan Z, Li Q, Luo S, Liu Z, Luo D, Zhang B, Zhang D, Rao P and Xiao J: PPAR $\gamma$ and Wnt signaling in adipogenic and osteogenic differentiation of mesenchymal stem cells. Curr Stem Cell Res Ther 11: 216-225, 2016.

19. Harsløf T, Tofteng CL, Husted LB, Nyegaard M, Børglum A, Carstens M, Stenkjær L, Brixen K, Eiken P, Jensen JE, et al: Polymorphisms of the peroxisome proliferator-activated receptor $\gamma$ (PPAR $\gamma$ ) gene are associated with osteoporosis. Osteoporos Int 22: 2655-2666, 2011.

20. Muruganandan S, Parlee SD, Rourke JL, Ernst MC, Goralski KB and Sinal CJ: Chemerin, a novel peroxisome proliferator-activated receptor gamma (PPARgamma) target gene that promotes mesenchymal stem cell adipogenesis. J Biol Chem 286: 23982-23995, 2011.

21. Mattern A, Zellmann T and Beck-Sickinger AG: Processing, signaling, and physiological function of chemerin. IUBMB Life 66: 19-26, 2014.

22. De Henau O, Degroot GN, Imbault V, Robert V, De Poorter C, Mcheik S, Galés C, Parmentier M and Springael JY: Signaling properties of chemerin receptors CMKLR1, GPR1 and CCRL2. PLoS One 11: e0164179, 2016.

23. Helfer G and Wu QF: Chemerin: A multifaceted adipokine involved in metabolic disorders. J Endocrinol 238: R79-R94, 2018.

24. Kennedy AJ and Davenport AP: International union of basic and clinical pharmacology CIII: Chemerin receptors CMKLR1 (Chemerin1) and GPR1 (Chemerin2) nomenclature, pharmacology, and function. Pharmacol Rev 70: 174-196, 2018.

25. Zhao H, Yan D, Xiang L, Huang C, Li J, Yu X, Huang B, Wang B, Chen J, Xiao T, et al: Chemokine-like receptor 1 deficiency leads to lower bone mass in male mice. Cell Mol Life Sci 76: 355-367, 2019.

26. Ramos-Junior ES, Leite GA, Carmo-Silva CC, Taira TM, Neves KB, Colón DF, da Silva LA, Salvador SL, Tostes RC, Cunha FQ and Fukada SY: Adipokine chemerin bridges metabolic dyslipidemia and alveolar bone loss in mice. J Bone Miner Res 32: 974-984, 2017. 
27. Muruganandan S, Govindarajan R, McMullen NM and Sinal CJ: Chemokine-like receptor 1 is a novel Wnt target gene that regulates mesenchymal stem cell differentiation. Stem Cells 35: 711-724, 2017.

28. Muruganandan S, Roman AA and Sinal CJ: Role of chemerin/CMKLR1 signaling in adipogenesis and osteoblastogenesis of bone marrow stem cells. J Bone Miner Res 25: 222-234, 2010

29. Goralski KB, McCarthy TC, Hanniman EA, Zabel BA, Butcher EC, Parlee SD, Muruganandan S and Sinal CJ Chemerin, a novel adipokine that regulates adipogenesis and adipocyte metabolism. J Biol Chem 282: 28175-28188, 2007.

30. An Q, Wu D, Ma Y, Zhou B and Liu Q: Suppression of Evi1 promotes the osteogenic differentiation and inhibits the adipogenic differentiation of bone marrow-derived mesenchymal stem cells in vitro. Int J Mol Med 36: 1615-1622, 2015.

31. Dominici M, Le Blanc K, Mueller I, Slaper-Cortenbach I, Marini F, Krause D, Deans R, Keating A, Prockop DJ and Horwitz E: Minimal criteria for defining multipotent mesenchymal stromal cells. The international society for cellular therapy position statement. Cytotherapy 8: 315-317, 2006.

32. Schmittgen TD: Real-time quantitative PCR. Methods 25 383-385, 2001

33. Bedi O, Dhawan V, Sharma PL and Kumar P: Pleiotropic effects of statins: New therapeutic targets in drug design. Naunyn Schmiedebergs Arch Pharmacol 389: 695-712, 2016.

34. Roh SG, Song SH, Choi KC, Katoh K, Wittamer V, Parmentier M and Sasaki S: Chemerin-a new adipokine that modulates adipogenesis via its own receptor. Biochem Biophys Res Commun 362 1013-1018, 2007

35. Gao K, Shen Z, Yuan Y, Han D, Song C, Guo Y and Mei X: Simvastatin inhibits neural cell apoptosis and promotes locomotor recovery via activation of $\mathrm{Wnt} / \beta$-catenin signaling pathway after spinal cord injury. J Neurochem 138: 139-149, 2016.

36. Robin NC, Agoston Z, Biechele TL, James RG, Berndt JD and Moon RT: Simvastatin promotes adult hippocampal neurogenesis by enhancing Wnt/ $\beta$-catenin signaling. Stem Cell Reports 2: 9-17, 2013.
37. Sawada N and Liao JK: Rho/Rho-associated coiled-coil forming kinase pathway as therapeutic targets for statins in atherosclerosis. Antioxid Redox Signal 20: 1251-1267, 2014.

38. Rikitake Y and Liao JK: Rho GTPases, statins, and nitric oxide. Circ Res 97: 1232-1235, 2005.

39. Rourke JL, Dranse HJ and Sinal CJ: CMKLR1 and GPR1 mediate chemerin signaling through the RhoA/ROCK pathway. Mol Cell Endocrinol 417: 36-51, 2015.

40. Tang QQ, Otto TC and Lane MD: Mitotic clonal expansion: A synchronous process required for adipogenesis. Proc Natl Acad Sci USA 100: 44-49, 2003.

41. Zanette DL,Lorenzi JC, Panepucci RA, Palma PV, Dos Santos DF, Prata KL and Silva WA Jr: Simvastatin modulates mesenchymal stromal cell proliferation and gene expression. PLoS One 10: e0120137, 2015.

42. Li Y, Müller AL, Ngo MA, Sran K, Bellan D, Arora RC, Kirshenbaum LA and Freed DH: Statins impair survival of primary human mesenchymal progenitor cells via mevalonate depletion, NF- $\kappa \mathrm{B}$ signaling, and Bnip3. J Cardiovasc Transl Res 8: 96-105, 2015.

43. Weivoda MM and Hohl RJ: Geranylgeranyl pyrophosphate stimulates PPAR $\gamma$ expression and adipogenesis through the inhibition of osteoblast differentiation. Bone 50: 467-476, 2012.

44. Nicholson AC, Hajjar DP, Zhou X, He W, Gotto AM Jr and Han J: Anti-adipogenic action of pitavastatin occurs through the coordinate regulation of PPARgamma and Pref-1 expression. $\mathrm{Br}$ J Pharmacol 151: 807-815, 2007.

45. Rourke JL, Muruganandan S, Dranse HJ, McMullen NM and Sinal CJ: Gprl is an active chemerin receptor influencing glucose homeostasis in obese mice. J Endocrinol 222: 201-215, 2014.

This work is licensed under a Creative Commons Attribution-NonCommercial-NoDerivatives 4.0 International (CC BY-NC-ND 4.0) License. 